\title{
SinONIMIZAÇões EM LoNCHocarpus Kunth (Leguminosae - Papilionoideae - Millettieae)
}

\author{
Ana Maria Goulart de Azevedo Tozzi ${ }^{1}$ \& Marcos José da Silva ${ }^{2}$
}

\section{Resumo}

(Sinonimizações em Lonchocarpus Kunth (Leguminosae - Papilionoideae - Millettieae) Baseado na análise de espécimes, incluindo coleções-tipo, quatro nomes são sinonimizados aqui: Lonchocarpus albiflorus Hassl. (= L. leucanthus Burkart) sob L. campestris Mart. ex Benth., L. ernesti Harms sob L. margaritensis Pittier, Robinia nicou Aubl. sob L. latifolius (Willd.) DC. e L. ehrenbergii Urb. sob L. rubiginosus Benth. A lectotipificação de L. campestris e L. ehrenbergii também é proposta.

Palavras-chave: Taxonomia, nomenclatura, sinônimos novos, lectótipification.

\section{Abstract}

(Synonymies in the Lonchocarpus Kunth (Leguminosae - Papilionoideae - Millettieae)). Based on the analysis of abundant specimens, including type-collections, four names are synonymized here: Lonchocarpus albiflorus Hassler (= L. leucanthus Burkart) under L. campestris Mart. ex Benth., L. ernesti Harms under L. margaritensis Pittier, Robinia nicou Aubl. under L. latifolius (Willd.) DC., and L. ehrenbergii Urb. under L. rubiginosus Benth. The lectotype of $L$. campestris and L. ehrenbergii is also proposed.

Key words: Taxonomy, nomenclature, new synonyms, lectotypification.

\section{INTRODUÇÃO}

Lonchocarpus com aproximadamente 120 espécies é um dos mais diversificados e complexos dentre os gêneros da tribo Millettieae (Schrire 2005). Apresenta distribuição predominantemente neotropical, sendo um importante componente das formações florestais da América Central e do Sul. Inclui espécies arbóreas, de folhas alternas com folíolos opostos ou subopostos, flores arranjadas em panículas, pseudoracemos ou pseudopanículas, terminais ou axilares, estames 10, pseudomonadelfos, tubo estaminal com margens calosas ou não na base e gineceu 1-10-ovulado, além de frutos em sua maioria indeiscentes (Tozzi 1989).

Após seu estabelecimento por Kunth (1823), este gênero recebeu importantes contribuições a sua sistemática, dentre as quais se ressaltam as de Bentham (1860, 1862) Pittier (1917), Geesink (1981) e Tozzi
(1989). Entre estes trabalhos, Tozzi (1989) forneceu uma revisão do estado do conhecimento do gênero à época, adotando uma circunscrição mais restrita para o mesmo, devido à exclusão das espécies americanas de Derris e de Lonchocarpus subg. Phacelanthus (=sect. Fasciculati), que foram transferidas para Deguelia, e a inclusão das espécies de Muellera. A autora reconheceu 23 espécies ocorrentes no Brasil, diagnosticadas principalmente pelo tipo e disposição das inflorescências, pela presença de pontuações translúcidas nos folíolos, pelo número de folíolos, tamanho das flores, presença de calosidades ou apêndices no estandarte, além de comprimento da unguícula das pétalas e tricomas em ambas as faces dos folíolos.

Após este tratamento taxonômico, há na literatura alguns trabalhos envolvendo a descrição de novos táxons (Mattos 1988;

Artigo recebido em 07/2006. Aceito para publicação em 12/2006.

${ }^{1}$ Universidade Estadual de Campinas, Instituto de Biologia, Departamento de Botânica, CP 6109, 13083-970, Campinas, São Paulo, Brasil

${ }^{2}$ Programa de Pós-Graduação em Biologia Vegetal, Universidade Estadual de Campinas/UNICAMP (CNPq nº 140609/2006-7)

${ }^{1}$ Autor para correspondência: anatozzi@ unicamp.br

Apoio financeiro: FAPESP (processo 80/1289-9), CNPq (processo 40.0342/82). 
Poppendieck 1992; Sousa 1986, 1999, 2005; Tozzi 1995), reavaliação da circunscrição de algumas espécies (Fortunato \& Palese 1997, Tozzi 1992) e análise de caracteres específicos como subsídio à classificação infragenérica (Teixeira et al. 2000).

Com base em estudos taxonômicos em desenvolvimento com espécies de Lonchocarpus e nos resultados propostos por Tozzi (1989), a circunscrição de quatro espécies, incluindo a revisão de seus sinônimos, é aqui estabelecida*.

\section{Resultados e Discussão}

Lonchocarpus campestris Mart. ex Benth., J. Linn. Soc., Bot. 4, Suppl. 95. 1860. Tipo: BRASIL. MINAS GERAIS: "in caatingas ad Rio São Francisco", s.d., C.F.P. Martius 13797 (lectótipo M! aqui designado).

L. leucanthus Burk., Darwiniana 4: 326. 1942.

L. albiflorus Hassl., Bull. Herb. Boissier, sér. 2, 7: 166. 1907, non G. Don. 1832. Tipo: PARAGUAI. "In regione fluminis Yhii in campis pr. San Joaquim”, XI.1905, Hassler 9656 (holótipo G!), syn. nov.

L. albiflorus f. parviflora Hassl., Bull. Herb. Boissier, sér. 2, 7: 167. 1907. Tipo: PARAGUAI. Yeruti, Serra da Maracayú, XII.1898-1899, Hassler 5754 (holótipo G!; isótipos A, BM!, G!, K!, NY, P!, S!), syn. nov.

L. microphyllus Glaz., Bull. Soc. Bot. France 53: 150. 1906, nom. nud.

L. nitidus auct. non Bentham: Chodat \& Hassl., Bull. Herb. Boissier, sér. 2, 4: 893. 1904.

Árvore, 4-8(20) m alt.; ramos lenticelados; estípulas caducas. Folhas 7 ou 9folioladas; pecíolo e ráquis pubescentes, ca. $2,5 \mathrm{~cm}$ e 2,5 $-5 \mathrm{~cm}$ compr., respectivamente; folíolos 1,5-5×1-2 cm, elípticos, ápice agudo,

\footnotetext{
* Os resultados foram obtidos da análise de exsicatas, incluindo coleções-tipo, provenientes dos herbários B, BHMH, BM, C, CEPEC, COL, FR, HB, IAC, IJ, L, LE, M, MBM, MG, NY, K, P, PACA, R, SP, U, UEC, US (siglas segundo Holmgren et al. 1990).
}

base atenuada a arredondada, pubescentes em ambas as faces. Pseudo-racemos com flores geminadas, 2,5-9 cm compr., axilares, multifloros, menores ou iguais às folhas, ou subfasciculados em ramos curtos, pubescentes, pedúnculo ca. 2,5 cm compr.; brácteas ovais, tomentosas; pedicelo 2-4 mm compr.; bractéolas ca. $1 \mathrm{~mm}$ compr., situadas na metade superior do pedicelo, linear-lanceoladas, caducas; cálice 4-5 mm compr., campanulado, tomentoso a seríceo, lacínios 4, agudos, ciliados, vexilar 1, largo-triangular, emarginado, carenais 3 , triangulares, $1-3 \times 1-2 \mathrm{~mm}$; corola 6-10 mm compr., geralmente branca; estandarte oval-orbicular, ápice emarginado, base subcordada, unguícula curta, face externa serícea; asas oblongo-falcadas, subauriculadas, unguiculadas, ápice seríceo; pétalas da quilha obovais, ápice seríceo; ovário curto-estipitado, seríceo-viloso; óvulos 5-6; estilete pubescente. Fruto 3-4 × 1,1-1,4 cm, compresso, oblongo ou elíptico, ápice agudo a acuminado, base atenuada, curto-estipitado, margens nerviformes, velutino; sementes 1-3, reniformes.

Material selecionado: BRASIL. BAHIA: Jequié, 16.X.1975, Hage 110 (CEPEC); Senhor do Bonfim, 26.II.1974, Harley 16377 (CEPEC, K); Tamburi, X.1906, Ule 7279 (K, L). CEARÁ: Meruoca, alto de Araripe, II.1860, Allemão \& Cysneiros 422 (R); ESPÍRITO SANTO: Colatina, Rio Doce, 1.XII.1943, J.G. Kuhlmann 6577 (UEC). MINAS GERAIS: Caldas, 14.XII.1847 (25.XII.1848 ou 12.II.1849), Regnell III 466, (C, K, LE, M, P, R, U); Conceição das Pedras, 25.XI.1967, J. Mattos 15205 \& N. Mattos (SP); "Tabuleiro verflug Gayes, in campo", s.d., $C$. Martius 13798 (síntipo de L. campestris, M); Coronel Pacheco, Estação Experimental, 20.XII.1945, E.P. Heringer 2186 (SP). PARANÁ: Foz do Iguaçu, Rio Ocuí, 7.XII.1969, G. Hatschbach 23145 (BM, C, HB, MBM). RIO GRANDE DO SUL: Nova Petrópolis, I.1943, Rambo 11012 (PACA); Osório, 14.XII.1949, Rambo 44739 (K, PACA). SANTA CATARINA: Campo Erê, 7.XII.1964, L.B. Smith \& Klein 13844 (K, US). SÃO PAULO: Barueri, 9.XII.1926, Hoehne 19068 (SP). ARGENTINA. SANTO TOMÉ: Província de Corrientes, Colonia Garabí, 3.XII.1970, Krapovickas et al. 17026 (C, IAC, P). PARAGUAI. Alto Paraná, 1910-1910, Fiebrig 6028 (BM, K, L). 
O nome Lonchocarpus leucanthus foi proposto por Burkart (1942) para substituir o nome $L$. albiflorus, estabelecido por Hassler (1907), que é um homônimo posterior de uma espécie da África tropical descrita por Don (1832). Ele foi amplamente utilizado na literatura botânica, especialmente na região sul da América do Sul. A análise das coleçõestipo de L. leucanthus e L. campestris evidenciou dois extremos de variação morfológica, mas o exame de uma quantidade maior de material oriundo da área compreendida entre o Nordeste do Brasil e a Argentina mostrou uma variação contínua nos estados de caráter diagnósticos, o que indicou que ambas são co-específicas, uma vez que as diferenças observadas em L. leucanthus e que a tornavam distinta de L. campestris, como na maior ou menor densidade de indumento nos ramos e pecíolo, no comprimento dos lacínios do cálice, número de óvulos do ovário e pilosidade do estilete, são variáveis. Burkart (1942), ao propor L. leucanthus, citou que o mesmo possuía flores com ovário 6-ovulado, no entanto, após a execução de cortes longitudinais em flores de $L$. campestris em diferentes estádios de desenvolvimento, notouse que o número de óvulos desta espécie varia de 4-6, embora sejam verificadas 1-3 sementes quando no estádio de fruto.

Espécie sul-americana (Argentina, Brasil e Paraguai). No Brasil cresce principalmente nas formações florestais das Regiões Nordeste (Bahia, Ceará, Paraíba), Sudeste (Minas Gerais, Espírito Santo, São Paulo) e Sul (Paraná, Rio Grande do Sul, Santa Catarina).

Lonchocarpus latifolius (Willd.) DC., Prodr. 2: 260. 1825. Derris latifolia (Willd.) Ducke, Bolm. Téc. Inst. Agron. N. 18: 195. 1949. Amerimnum latifolium Willd., Spec. Pl. 3: 909. 1802. Tipo: COLÔMBIA. CARTAGENA: s.d., Willdenow 13088 (holótipo B!; foto holótipo UEC!, US!)

Robinia nicou Aubl., Pl. Guian. 2: 771, pl. 308. 1775. Tipo: GUIANA FRANCESA. "Orapu prope praedium domini Budet", s.d., Aublet s.n. (holótipo BM!; foto holótipo NY!, US!, UEC!), syn nov.
L. swartzii DC., Prodr. 2: 261. 1825. $R$. sepium Sw., Fl. Ind. Occid. 3: 1258. 1806, non Jacq. Tipo: AMÉRICA CENTRAL, s.d., Swartz s.n. (holótipo C!)

L. pentaphyllus (Poir.) DC., Prodr. 2: 259. 1825. Dalbergia pentaphylla Poir., Encycl. Méth. Bot. Suppl. 2: 445. 1811. Tipo: PORTO RICO. s.l., s.d., Ledru s.n. (holótipo P!)

L. heptaphyllus (Poir.) DC., Prodr. 2: 259. 1825. D. heptaphylla Poir., Encycl. Méth. Bot. Suppl. 2: 446. 1811. Tipo: REPÚBLICA DOMINICANA. SANTO DOMINGO: s.l., s.d., Poiteau s.n. (holótipo $\mathrm{P}$; isótipo $\mathrm{G}$ !)

L. discolor Huber, Bol. Mus. Para. 3: 421. 1902. Tipo: BRASIL. PARÁ: Breves, "furo Mucujubim", Guedes s.n. (holótipo MG!; isótipos BM!, US!; foto isótipo F!).

Árvore, 3-8 m alt.; ramos estriados, lenticelados, glabrescentes; estípulas caducas. Folhas 5 ou 7 (9)-folioladas; pecíolo (4) 8,5-12 cm compr., ráquis $(2,5)$ 5-8 cm compr.; peciólulo 5-8 mm compr., rugoso; folíolos 8-16 (24) × 3-8 (12) cm, oval-lanceolados a elípticos, ápice acuminado, mucronado, pubescentes em ambas as faces. Pseudo-racemos com braquiblastos curtos e bifloros, menores que as folhas, axilares, densifloros, eixo lenhoso, estriado, criso-tomentoso; brácteas ovais, ca. $1 \mathrm{~mm}$ compr., persistentes; pedicelo $2-$ $4 \mathrm{~mm}$ compr., tomentelo; bractéolas 2 no ápice do pedicelo, menores que $1 \mathrm{~mm}$ compr., oval-oblongas, tomentelas, persistentes; cálice 2-3 $\mathrm{mm}$ compr., crateriforme, base atenuada, tomentoso; lacínios 5, deltóides, obsoletos; corola 6-8 $\mathrm{mm}$ compr., púrpura a vermelho-escura, raramente branca (Cuba e Jamaica); estandarte suborbicular, base truncada, bicalosa, ápice arredondado a emarginado, criso-seríceo na região centro-apical, curtounguiculado; asas subfalcadas, oblongas, subauriculadas, glabrescentes; quilha semioboval, serícea na nervura principal; ovário linear, subséssil, denso-seríceo, óvulos $2-$ 4. Fruto 5-8 × 2-2,3 cm, elíptico, curtoestipitado (estipe ca. $3 \mathrm{~mm}$ compr.), 
compresso, ápice e base atenuados, margens nerviformes, papiráceos, seríceos, constritos ou não na região das sementes; sementes 1-3, subarredondadas, compressas.

Material selecionado: BRASIL. MINAS GERAIS: Belo Horizonte, 12.XII.1942, Oliveira 1184 (BHMH); Pará: Mosqueiro, 4.XI.1967, Pires \& N.T. Silva 11266 (IAN); RIO DE JANEIRO: Rio de Janeiro, IV.1969, Emygdio 2654 (R); SÃO PAULO: Campinas, 2.III.1978, Mathes 10071 (UEC). BELIZE: Belize, Distrito de Cayo, 26.VI.1973 Gentry 8613 (C). COLÔMBIA: ANTIOQUIA, Turbo, 24.III.1945, Haught 4538 (COL, K, US). COSTA RICA: Provincia de Puntarenas, Playa Blanca, Rincon de Osa, 16.II.1974, Liesner 2185 (IJ). CUBA: Monte Verde, 1860-1864, Wright 142 (P). DOMINICA: Prince Rupers, IV.1882, Eggers 743 (FR, L, P). GUIANA: Dist. Northwest, Koriabo, Rio Barima, 712.IX.1934, Archer 2505 (US). JAMAICA: Parish Negril, 7.X.1975, Proctor 35350 (IJ). VENEZUELA: DISTRITO FEDERAL: Los Caracas, Hacia caruo, 1.V.1969, Aristeguieta 7104 (US).

Após o estabelecimento da identidade de Robinia nicou e Lonchocarpus latifolius, o epíteto nicou teria prioridade por ser o mais antigo. No entanto, devido a interpretações equivocadas da identidade do nome proposto por Aublet (1775), que foi utilizado para Deguelia utilis (A.C. Sm.) A.M.G. Azevedo e para L. nitidulus Benth., entre outras, uma nova combinação é impossível, pela existência de $L$. nicou DC., táxon pertencente a Deguelia e que, segundo De Candolle (1825: 261), se diferencia por apresentar inflorescências maiores que as folhas e face abaxial dos folíolos com tricomas seríceos. Portanto, a espécie em questão deve ser chamada de L. latifolius (Willd.) DC. Muitos autores atribuem a autoria desta combinação a Humb., Bonpl. \& Kunth ou a Kunth (1823), mas a transferência desta espécie para Lonchocarpus apenas foi sugerida por estes, sendo efetivada por De Candolle (1825).

Poiret (1816) transferiu Amerimnum latifolium para Pterocarpus, com o sinônimo A. pinnatum, descrita por Jacquin (1763), baseada em uma planta de Cartagena. No entanto, nem Kunth (1823) nem De Candolle (1825) concordaram com o procedimento de
Poiret (1816). Kunth (1823) comentou que $A$. pinnatum difere de $A$. latifolium e de Lonchocarpus. De Candolle (1825) tratou-a como L. amerimnum. Observando-se a prancha fornecida por Jacquin (1763) nenhuma conclusão pôde ser tirada, porém, através da análise do protólogo da espécie, achamos que esta talvez se enquadre melhor em Platymiscium, pelas folhas opostas.

Lonchocarpus pentaphyllus e L. heptaphyllus, descritas por Poiret (1811) e que diferem principalmente no número de folíolos, foram sinonimizadas em L. latifolius por Bentham (1860). Grisebach (1864) considerou L. pentaphyllus e L. heptaphyllus distintas de L. latifolius, e acrescentou L. swartzii (= Robinia sepium) em sua sinonimia. $\mathrm{O}$ binômio Robinia scandens Willd. (Willdenow 1802) foi sinonimizado com L. nicou, por De Candolle (1825), mas este nome é supérfluo, pois foi baseado no mesmo tipo de $R$. nicou. A espécie descrita por Huber (1902), baseada numa planta amazônica e nomeada L. discolor, foi sinonimizada em L. latifolius por Ducke (1949).

Existe certa discordância entre os autores sobre o nome correto da espécie, se $L$. latifolius DC. ou L. pentaphyllus (Poir.) DC. Johnston (1949) justificou que "o nome dúbio L. latifolius deveria ser substituído pelo bem tipificado L. pentahphyllus". No entanto, pelos comentários de Johnston (1949) nota-se que ele não observou o material tipo da espécie (Willdenow 13088), pois o mesmo comentou que Amerimnum latifolius foi baseada na ilustração e descrição de uma árvore coletada por Jacquin, que corresponde a $A$. pinnatum. A exsicata de $A$. pinnatum seguramente não é a presente espécie e talvez não seja nem um membro de Lonchocarpus, como já mencionado. A dubiedade do epíteto específico latifolius foi estabelecida por Poiret (1816), como já discutido acima, quando ele transferiu A. latifolius para o gênero Pterocarpus, incluindo $A$. pinnatum. De Candolle (1825) excluiu este sinônimo, $A$. pinnatum, de $A$. latifolium quando o transferiu para Lonchocarpus. O tipo de A. latifolium 
corresponde a esta espécie e, portanto este deve ser o binômio válido para a mesma.

Ocorre desde a América Central (Belize, Costa Rica, Honduras, Guatemala, Nicaragua e Panamá), incluindo as Antilhas (Cuba, Jamaica, República Dominicana, Guadelupe, Martinica e Trinidad e Tobago) até o Norte da América do Sul (Brasil, Colômbia, Guiana, Guiana Francesa, Suriname e Venezuela). No Brasil é registrada como nativa para os estados do Amapá, Pará e São Paulo e como cultivada em Minas Gerais e Rio de Janeiro (Bentham 1860; Ducke 1925, 1949). Cresce preferencialmente em margens inundadas de rios do estuário e do litoral.

Lonchocarpus margaritensis Pittier, Contrib. U.S. Nat. Herb. 20: 91. 1917. Tipo: VENEZUELA. "El Valle, Ilha de Margarita", 7.VII.1903, Jonston 23 (holótipo NY!; isótipos $\mathrm{C}$ !, F!).

L. ernesti Harms, Reprium Nov. Spec. Regni Veg. 17: 321. 1921. Derris ernesti (Harms) Ducke, Bolm. Téc. Inst. Agron. N. 18:196. 1949. Tipo: BRASIL. RORAIMA. Surumu, Serra do Sol, Ule 8167 (holótipo K!; isótipos L!, US!; foto isótipos NY! C!, F!), syn. nov.

Árvore 10-20 m alt.; ramos estriados, lenticelados; estípulas triangular-lanceoladas, caducas. Folha 7 ou 9 (5 ou 11)-foliolada; pecíolo 4-6 cm compr., estriado, pubescente; ráquis 5,5-9,5 cm compr.; peciólulo 5-7 $\mathrm{mm}$ compr., tomentoso; folíolos 5,5-9 × 2-5 cm, elípticos, oblongo-lanceolados a obovais; ápice agudo a mucronado; base arredondada a oblíqua; cartáceos a subcoriáceos; pubescentes em ambas as faces. Pseudo-racemos ou pseudopanículas 4,5-11 cm compr., indumentados, com braquiblastos curtos e bifloros, axilares, multifloros; brácteas até 3 mm compr., caducas; bractéolas ca. $2 \mathrm{~mm}$ compr., linear-lanceoladas, viloso-tomentosas, caducas; cálice 3-4 mm compr., cupuliforme, castanho a avermelhado; lacínios 4, ciliados, vilosos a lanuginosos; vexilar arredondado, largo, ciliado, viloso-lanuginoso, carenais 3, agudos, central lanceolado, seríceo-tomentoso na face interna; corola 8-12 mm compr., rosada, lilás a avermelhada, filetes brancos, anteras amarelas; asas e pétalas da quilha marrom-avermelhadas; hipanto pouco desenvolvido; estandarte orbicular-oval; ápice retuso; base subauriculada, unguícula curta e carnosa, seríceo na face externa, na interna esparsamente curto-pubescente, com um tufo na região centro-basal acima da unguícula; asas falcadas, glabrescentes, levemente plicadas, unguiculadas; pétalas da quilha falcadas, ungüiculadas, seríceas externamente; ovário velutino; óvulos 4, estilete glabro, esparso ciliado na base. Fruto 9-12,5 × 2,8-3,5 cm quando monospermo, 11$16,5 \mathrm{~cm}$ compr. quando bispermos, elíptico a semi-elíptico, longo-estipitado, constrito ou não na região das sementes; base atenuada, margem nerviforme, cartáceo a subcoriáceo, reticulado e tomentoso.

Material selecionado: BRASIL. RORAIMA: Boa Vista, 23.VIII.1943, Ducke 1393 (K, RB, US); São Marcos, Posto dos Índios entre os rios Tacutu e Uiraricuera, 3.IX.1951, Black 51-13260, (COL, K, US); Alto Surumu, Serra da Memória próximo a Fazenda Triunfo, 29.IV.1980, I. A. Rodrigues et al. 688 (UEC). GUIANA. Extremidade Oeste das montanhas Kanaku, no curso do Rio Takutu, 422.III.1938, A. C. Smith 3225 (B, K, P). VENEZUELA. CANTAURA: 28.IV.1950, Gines 4393 (US); ARAGUA: La Victoria, 1856/1857, Fendler 1861 (K, fotografia NY 2733, C, F);

Lonchocarpus margaritensis foi estabelecido por Pittier (1917), que mencionou que a espécie difere de L. velutinus Benth. principalmente pelos caracteres florais e que se apresenta relacionada morfologicamente com as espécies da seção Spongopteri. Harms (1921) descreveu L. ernesti a partir de material florido. Analisando os protólogos e as coleções-tipo de L. margaritensis e de L. ernesti, bem como a distribuição geográfica das mesmas e material adicional, constatou-se uma grande uniformidade morfológica, o que levou-nos a inclusão de L. ernesti sob sinônimo de L. margaritensis.

A coleção Fendler 1861 foi utilizada por Bentham (1860) como um dos sintipos de L. macrocarpus. No entanto, este nome foi estabelecido a partir de materiais de diferentes espécies. Embora Pittier (1944) 
tenha designado a coleção Fendler 1861 como sendo o lectótipo da espécie, Sousa (1990) argumentou que a escolha deste lectótipo foi automática, uma vez que Pittier trabalhava com a Flora da Venezuela, e rejeitou a lectotipificação proposta por Pittier (1944), designando como lectótipo a coleção New Spain: Herb. Pavon s.n, a qual se encontra depositada no herbário de Genebra (G), uma espécie diferente da aqui tratada. Neste trabalho, Sousa (1990) informou ainda que o exemplar Fendler 1861 corresponde a $L$. hedyosmus Miq., mas a correta identificação deste espécime é L. margaritensis.

Pittier $(1917,1944)$ posicionou o táxon na seção Spongopteri, mas seu melhor posicionamento é na seção Densiflori, próximo a L. spiciflorus Benth. e L. latifolius, por apresentar inflorescências pseudopaniculadas, entre outras características.

Espécie com distribuição restrita ao norte da América do Sul, no Brasil (Roraima), Guiana e Venezuela. Cresce em vegetação campestre, savanas arbóreas densas ou de altitude e florestas, sob solo arenoso ou rupestre, próximo a rios em geral entre 100 a $340 \mathrm{~m}$ de altitude.

Lonchocarpus rubiginosus Benth., J. Linn. Soc., Bot. 4, Suppl. 92. 1860. Tipo: GUIANA FRANCESA. s.l., s.d., Aublet s.n. (holótipo BM!)

L. ehrenbergii Urb., Symb. Antill. 3: 283. 1902. Tipo: HAITI. "prope Portum Principis", s.d., Faegen 201 (lectótipo C!, designado aqui, isolectótipos NY!, GH!, LE!), syn. nov.

Árvore ca. $5 \mathrm{~m}$ alt.; ramos lenticelados; estípulas caducas. Folhas (5) 7, 9, 11(13)folioladas; pecíolo 0,8-2cm compr.; raque 0,4-7 cm compr., ambos sulcados, seríceos; folíolos 1,5$4 \times 0,5-0,7 \mathrm{~cm}$, elípticos a obovais, ápice agudo, mucronulado, base cuneada a obtusa, face adaxial glabra, abaxial serícea. Pseudo-racemos com flores geminadas, 3-6 cm compr., eixo seríceo-ferrugíneo, axilares, brácteas de 1a. ordem caducas; brácteas de $2^{\mathrm{a}}$ e $3^{\mathrm{a}}$ ordem, ca. 1 mm compr., ovais, agudas, seríceas; pedicelo ca. 2 mm compr.; bractéolas ca. 1 mm compr., situadas na base do cálice, ovais, agudas, pontuadas; cálice 2-2,5 $\mathrm{mm}$ compr., campanulado, seríceo, lacínios 4, agudos, vexilar 1, largo-triangular, carenais 3, raso-trinagulares; estandarte orbicular, ápice emarginado, convoluto, base atenuada, bi-apendiculado, bi-caloso; unguícula curta, face externa serícea na porção apical; asas oblongo-falcadas, ápice agudo, sagitadas na margem vexilar, seríceas no ápice, pontuadas; pétalas da quilha ca. 5,2 mm compr., ungüicula curta (ca. $2 \mathrm{~mm}$ ), falcadas, ápice agudo, pontuadas, plicadas próximo à base; ovário ca. $5 \mathrm{~mm}$ compr., linear, curto-estipitado (estipe ca. $1 \mathrm{~mm}$ compr.), seríceo; óvulos 3-4; estilete glabro. Fruto 2,5-3 $\times 0,3-0,4 \mathrm{~cm}$, linear, ápice arredondado a obtuso, base atenuada, estipitado, margens nerviformes, seríceo.

Analisando as coleções-tipo e a distribuição geográfica de L. rubiginosus e L. ehrenbergii não se observou descontinuidade nem variações nos caracteres suficientes para que estas sejam tratadas como espécies distintas, tais como a forma, o número e a consistência dos folíolos, a forma das peças florais (estandarte, alas e pétalas da quilha), a forma dos tricomas e o tipo de indumento, a coloração e o comprimento dos frutos, além do comprimento e disposição das inflorescências. Diante disto, resolveu-se incluir L. ehrenbergii no conceito de L. rubiginosus.

A espécie possui folíolos em número de (5) 7, 9, 11 (13), elípticos a obovais de ápice agudo e mucronulado, base cuneada a obtusa, face adaxial glabra e abaxial ferrugíneopubescente, inflorescências com eixos bifloros e ovário pluriovulado, além de frutos compressos e estreito lineares. Sua distribuição estende-se desde a América Central (Haiti, Caribe, República Dominicana) até a América do Sul (Guiana Francesa).

Lonchocarpus rubiginosus foi descrito por Bentham (1860), baseando-se numa planta coletada por Aublet s.n. na Guiana e depositada no herbário British Museum (BM) sob o binômio não publicado, Robinia rubiginosa Aubl. O mesmo autor (Bentham 1860) comentou que a espécie possui folhas e flores semelhantes morfologicamente a L. parviflorus (América Central), diferindo pela forma e comprimento dos frutos. 
Lonchocarpus ehrenbergii foi descrito por Urban (1902) baseado na coleção Faegen 201, cujas amostras se encontram depositadas nos herbários NY, C, GH e LE. Analisando os espécimes, verificou-se que o exemplar depositado no herbário de Copenhagem (C) se apresenta em conformidade com o protólogo da espécie, sendo o mais completo e, portanto, o mesmo é aqui escolhido como lectótipo.

\section{REFERÊNCIAS BIBLIOGRÁFICAS}

Aublet, J. B. C. F. 1775 . Histoire des plantes de la Guiane Françoise. Pierre Françoies Didot jeune, Paris, 308p.

Bentham, G. 1860. Synopsis of Dalbergieae, a tribe of Leguminosae. Journal of the Linnean Society, Bot., 4 (suppl.): 1-128. 1862. Leguminosae. In: Martius, C. F. P. von; Eichler, A. W. \& Urban, I. (eds.), Flora brasiliensis. F. Fleischer, Lipsiae, 15(1): 277-286.

Burkart, A. 1942. Algunas leguminosas nuevas de la Flora Argentina. Darwiniana 4(2-3): 323-331.

De Candolle, A. P. 1825. Leguminosae. In: Candolle, A. P. (ed.). Prodromus systematis naturalis regni vegetabilis. Vol. 2. Treuttel \& Würtz, Paris. Pp. 93-423.

Don, G. 1832. A general system of gardening and botany. Vol. 2. Gilbert \& Rivington, London. Pp. 91-475.

Ducke, A. 1925. Plantes nouvelles ou peu connues de la région amazonienne III. Arquivos do Jardim Botânico do Rio de Janeiro 4: 1-90. 1949. Notas sobre a Flora Neotrópica - II As leguminosas da Amazônia brasileira. Boletim Técnico do Instituto Agronômico do Norte 28: 35-38.

Fortunato, R. H. \& Palese, R. 1997. Una nueva combinación en el género Lonchocarpus Kunth (Leguminosae-Millettieae): Lonchocarpus fluvialis (Lindm.) Fortunato \& Palese. Candollea 52: 509-511.

Geesink, R. 1981. Tephrosieae (Benth.) Hutch. In: Polhill, R. M. \& Raven, P. H. Advances in legume systematics. Vol. 2. Royal Botanic Gardens, Kew. Pp. 245-260.

Grisebach, A. H. R. 1864. Flora of the British West Indian Island. Vol. 1. Lovell Reeve \& co., London. Pp. 186-200.

Harms, H. 1921. Einge neue. Lonchocarpus Arten aus dem tropischen Amerika. Reprium nov. Spec. Regni Veg. 17: 320-325.

Hassler, E. 1907. Plantae paraguariemsis, novae vel minus cognitae. II - Deux Lonchocarpus nouveaux des campos du Paraguay. Bulletin de l'herbier Boissier, ser. 2, 7(3):161-173.

Holmgren, P. K.; Holmgren, N. H. \& Barnett, L. C. 1990. Index Herbariorum. 8ed. New York Botanical Garden, New York, 691p.

Huber, J. 1902. Materiais para a flora amazônica V. Boletim do Museu Paraense Emílio Goeldi 3: 421-422.

Jacquin, N. J. 1763. Selectarum stipium americanarum historia. Facsimile ed. 1971, Hafner Publishing, New York. Pp. 200-201.

Johnston, I. M. 1949. The Botany of San Jose Island (Gulf of Panama). Sargentia 8:154.

Kunth, C. S. 1823. Voyage de Humboldt et Bonpland. In: Humboldt, F. H. A. von, Bonpland, A. J. A. \& Kunth, K. S. Nova Genera et Species Plantarum, facsimile ed. 1963. J. Cramer, Weinhein, 6: 382-385.

Mattos, N. F. 1988. Lonchocarpus torrensis N. Mattos. Loefgrenia, Porto Alegre 92: 2-5.

Pittier, H. 1917. The middle American Species of Lonchocarpus. Contributions from the United States national herbarium 20(2): 37-93.

1944. Leguminosas de Venezuela I. Papilionáceas. Ministerio de Agricultura y Cría, 5: 96-105.

Poiret, J. L. 1811. Pterocarpus. In: Lamarck, M. Encyclopédie methodique Botanique. Vol. 5. H. Agasse, Paris. Pp. 445-447. 1816. Pterocarpus. In: Lamarck, M. Encyclopédie methodique Botanique. Vol. 5. H. Agasse, Paris. Pp. 610-611. 
Poppendieck, H. 1992. New taxa of Lonchocarpus (Fabaceae:Millettieae) from the Venezuelan Guayana. Novon 2: 53-57.

Schrire, B. D. 2005. Tribe Millettieae. In: Lewis, G. P.; Scrhire, B.; Mackinder, B. $\&$ Lock, M. Legumes of the world. Royal Botanic Gardens, Kew, 577p.

Sousa, M. 1986. Adiciones a las Leguminosas de la flora de Nicaragua. Annals of the Missouri Botanical Garden 73(4): 722-737. 1990. Adiciones a las Papilionadas de la flora de Nicaragua. Annals of the Missouri Botanical Garden 77: 573-577. 1999. Especie nueva de Lonchocarpus (Leguminosae) de Nicaragua y Costa Rica. Anales del Instituto de Biología, Universidad Nacional Autónoma de México, série Botánica 70(2): 137-140.

2005. Las especies del género

Lonchocarpus sect. Lonchocarpus
(Leguminosae, Papilionoideae: Millettieae) para a Bolivia. Novon 15: 590-598.

Tozzi, A. M. G. A. 1989. Estudos taxonômicos dos gêneros Lonchocarpus Kunth e Deguelia Aubl. no Brasil. Tese de Doutorado. Universidade Estadual de Campinas, Campinas.

1992. Lonchocarpus monilis (L.) Az.Tozzi, comb. nov. (Leguminosae Papilionoideae): considerações nomenclaturais e taxonômicas. Revista Brasileira de Botânica 15(2): 151-155.

1995. New species of Lonchocarpus (Leguminosae: Papilionoideae: Millettieae) from Brazil. Kew Bulletin 50(1): 173-177.

Teixeira, S. P.; Castro, M. M. \& Tozzi, A. M. G. A. 2000. Secretory cavities and pellucid dots in leaflets of Lonchocarpus (Leguminosae, Papilionoideae, Millettieae). Plant Systematic and Evolution 221: 61-68.

Urban, I. 1902. L. ehrenbergii Urb. Symbolae Antillarum 3(2): 283. 